\title{
Isolated hyperbilirubinemia as the manifestation of acute liver failure in a patient with acute myelogenous leukemia and COVID-19 infection
}

\author{
Nasrin Gholami (1),Vahid Sheykhzadeh Amzajerdi (1), Rahim Mehdioghli (1), \\ Hengameh Khadivi Heris (1), Mona Jahandideh Kazempour (2) \\ (1) Hematology Oncology Research Center, Tabriz University of Medical Sciences, Tabriz, Iran \\ (2) Deparment of Microbiology, Faculty of Medical Genetic, Science and Research Islamic Azad \\ University, Tehran, Iran.
}

This article is distributed under the terms of the Creative Commons Attribution Noncommercial License (CC BY-NC 4.0) which permits any noncommercial use, distribution, and reproduction in any medium, provided the original author(s) and source are credited.

\begin{abstract}
Acute liver failure is a rare but catastrophic syndrome. Liver function tests usually reveal cholestatic or hepatocellular pattern, but in this case, we report a previously undescribed manifestation of this condition as isolated hyperbilirubinemia. Our patient was a 50 years old male who was diagnosed with acute myelogenous leukemia (AML(M4)). The patient had no history of liver disease and had normal liver function tests at the time of admission. After chemotherapy treatment, the patient developed signs of acute liver failure and had a rapid demise. After further investigations, we found two possible causes. Firstly, liver involvement of acute myelogenous leukemia (AML) which after induction chemotherapy is a rare condition and has high mortality rates despite treatment. Secondly, acute liver injury due to COVID-19 infection.
\end{abstract}

Key Words: Isolated hyperbilirubinemia; acute myelogenous leukemia; acute liver failure; COVID-19.

Eur J Transl Myol 31 (3): 9817, 2021 doi: 10.4081/ejtm.2021.9817

\begin{abstract}
Acute liver failure usually manifests itself as clinical signs and symptoms or abnormal liver function tests with a hepatocellular or a cholestatic pattern. ${ }^{1}$ Isolated hyperbilirubinemia is a very rare manifestation to the best of our knowledge. Moreover, liver involvement with acute myelogenous leukemia (AML) has rarely been reported. Most reported cases of AML with liver involvement had an exacerbating disease course with a very poor outcome. ${ }^{2,3}$ Furthermore, it is known that viral infections play a major role in causing acute liver injury, especially in developing countries. ${ }^{1}$ We could not find any trace of hepatotropic viruses in this case, but we found a COVID-19 infection which could be a risk factor or a comorbidity of acute liver failure.
\end{abstract}

\section{Case presentation}

A 50 years old addicted (to edible opium) male with no significant past medical history was referred to our hematology department for investigations. The patient complained about a 2-months history of loss of appetite and 2-weeks of generalized ecchymotic and petechial skin lesions mostly on lower limbs. Laboratory analysis showed leukocytosis (WBC: 50000 / mL) with thrombocytopenia (Plt: 12000 / mL) and anemia (Hb: 11 $\mathrm{g} / \mathrm{dL})$. The patient was admitted to the hematology ward with a possible diagnosis of Leukemia. After admission, the patient underwent bone marrow biopsy and bone marrow aspiration (Figure 1, upper left panel) which confirmed Acute Myelogenous Leukemia (M4, AML). On the 3rd day of admission, the induction chemotherapy with standard $7+3$ regimen (7 days Cytarabine 100 $\mathrm{mg} / \mathrm{m}^{2}, 3$ days Idarubicin $12 \mathrm{mg} / \mathrm{m}^{2}$ ) was started. WBC count decreased rapidly, and on the 6th day after admission, the patient went in a deep neutropenic state with an average Absolute Neutrophil Count of $50 / \mathrm{mL}$. The bone marrow continued to be suppressed throughout the disease course. Platelet count $(10000 / \mathrm{mL}$ on average) and hemoglobin levels (6-7 g/dL on average), remained low although the patient was receiving 10 units of platelets and 2 units of packed-cells daily.

On the 12th day after admission, the patient developed mild jaundice, which was visibly progressive, and subjectively the patient became more icteric every day. In addition, the Bilirubin levels, especially conjugated bilirubin started to rise but curiously AST and ALT levels remained normal. Albumin levels dropped and the patient slowly developed progressive pitting edema which later transformed into severe anasarca edema. The 

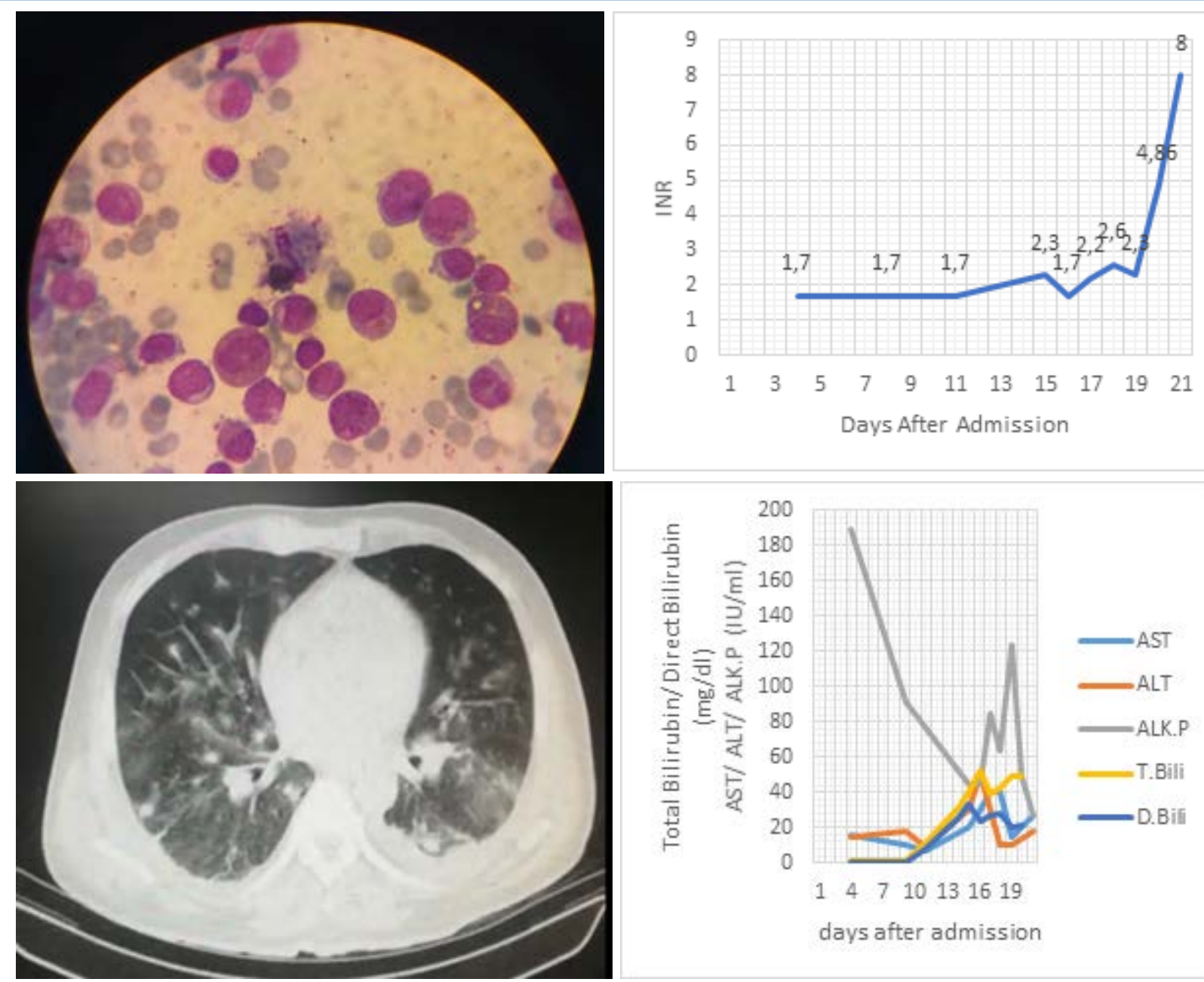

Fig 1. Upper left panel: Bone marrow aspiration. Myeloblasts in High-power field of microscope. There was nearly 40 percent myeloblasts in marrow of this patient. Upper right panel: International Normalized Ratio; Bottom left panel: Ground glass opacity in peripheral aspects of both lungs highly suggestive of COVID-19 pneumonia. Bottom right panel: D) AST, ALT, ALK.P, Total Bilirubin and Direct Bilirubin levels

administration of NAC was started. On the 16th day after admission, the patient developed signs of hepatic encephalopathy including Asterixis and altered sleep pattern so lactulose 30 cc TDS was prescribed. In addition, prothrombin time and INR began to elevate (Figure 1, upper right panel), subsequently, the infusion of 2 units of Fresh Frozen Plasma every 12 hours was started. Hbs-Ag, Hbs-Ab, HCV-Ab, HAV-Ab-IgM, HIV-Ab, CMV-Ab-IgM, EBV-Ab-IgM, HSV-I\&II-Ab, ANA, AMA, ASMA were checked and they were all negative. Imaging studies were suggestive of moderate hepatomegaly with diffuse parenchymal infiltration. Due to the patient's thrombocytopenia and elevated prothrombin time, liver biopsy was not performed.

On day 17 after admission, the $\mathrm{O}_{2}$ saturations dropped to $70 \%$ and a chest CT-Scan was requested and done, which was suggestive of COVID-19 infection (Figure 1. bottom left panel) and later the PCR test was positive for SARSCOV-2. On day 20, the patient was transported to the
Intensive Care Unit and unfortunately, 2 days later expired due to multiple organ failure. The patient's family did not consent for an autopsy so it was not performed. The detailed trends of laboratory analises are shown in (Figure 1, bottom right panel).

\section{Discussion}

Previous reports show either a cholestatic pattern or a transaminitis picture, ${ }^{1-4}$ but in this case, we are reporting a previously undescribed isolated hyperbilirubinemia as the presentation of the acute liver failure in the setting of AML liver involvement. On another aspect, the patient tested positive for SARS-COV-2 which raised some questions about the causality, triggers, and risk factors of acute liver failure. Considering the rapid progress of hepatic failure, we speculated that COVID-19 infection could play a role in exacerbating or triggering the condition. In a large U.S. cohort study, Phipps et al., ${ }^{5}$ demonstrated that acute liver injury is common in 
patients who test positive for SARS-COV-2, but it is most often mild. However, $6.4 \%$ of patients had a severe liver injury, with a severe disease course. ${ }^{5}$ A largely hepatocellular pattern of liver injury with few patients with elevated bilirubin and/or elevated alkaline phosphatase was observed. Significant hypoalbuminemia was seen, particularly among patients with severe liver injury. They additionally report that bilirubin was often normal even in extreme cases, ${ }^{5}$ unlike the situation of our patient. That cohort study shows a hepatocellular pattern as the manifestation of liver involvement in COVID-19 infection, ${ }^{5}$ which differs from our case's presentation, but it also demonstrates that liver injury is common among COVID-19 patients. The mechanism of liver injury remains largely unknown and is thought to be a combination of both direct virally-mediated effects as well as a result of the immune response., ${ }^{6,7}$ Viral infections are predominant causes of acute liver failure in developing countries, especially hepatitis A, B, and E viruses. ${ }^{1}$ It is clear that COVID-19 infection can also damage the liver tissue and in theory, it could at least worsen the progression of liver failure. However, it remains unclear if this infection could cause in the already susceptible liver to fail or it is a risk factor of fulminant hepatic failure. Isolated hyperbilirubinemia is a very rare presentation of the rare acute liver failure syndrome. In our investigations, we found two possible causes for this case. Firstly, AML involvement of the liver which is also uncommon. Secondly, COVID-19 infection which can cause liver injury. Or a combination of these two that eventually leads to this unique manifestation of this condition. In any case, future research with a large sample pool, case series, and longitudinal studies are required. Here are some of our recommended subjects for future research: i) isolated hyperbilirubinemia as oppose to cholestatic and hepatocellular patterns in acute liver failure; ii) liver involvement and acute liver failure in AML patients and the response to chemotherapy; iii) acute liver failure and it's risk factors in COVID-19 infection.

\section{List of acronyms}

ALF: Acute Liver Failure

ALK.P: Alkaline phosphatase

ALT: Alanine aminotransferase

AMA: Antimitochondrial Antibody

AML: Acute Myelogenous Leukemia

ANA: Antinuclear Antibody

ASMA: Anti-Smooth Muscle Antibody

AST: Aspartate aminotransferase

CMV: Cytomegalovirus

COVID-19: Coronavirus Disease of 2019

EBV: Epstein-Barr Virus

Hb: Hemoglobin

INR: International Normalized Ratio

NAC: N-Acetylcysteine

PCR: Polymerase Chain Reaction

Plt: Platelets

\section{Authors Contributions}

NG, VSA, RM, HKH, MJK participated in data collection, wrote the manuscript and reviewed literature. All authors critically reviewed final draft and approved the submitted typescript.

\section{Acknowledgments None.}

Funding None.

\section{Conflict of Interest}

The author declares no competing interests.

\section{Ethical Publication Statement}

We confirm that we have read the Journal's position on the issues involved in ethical publication and affirm that this report is consistent with those guidelines.

\section{Corresponding Author}

Nasrin Gholami, Hematology Oncology Research Center, Tabriz University of Medical Sciences, Tabriz, Iran. ORCID iD: 0000-0002-9546-0186

Email: dr.gholami_internist@yahoo.com

\section{E-mails and ORCID iD of Coauthors \\ Vahid Sheykhzadeh Amzajerdi: vahidsheykh94@gmail.com}

ORCID iD: 0000-0002-3154-6044

Rahim Mehdioghli: mehdioghloorahim@gmail.com ORCID iD: 0000-0001-5460-7383

Hengameh Khadivi Heris: hengame.khadiviheris@gmail.com

ORCID iD: 0000-0002-0501-7376

Mona Jahandideh Kazempour: monajahandidehkazempour@yandex.com

ORCID iD: 0000-0002-8673-0949

\section{References}

1. Sobotka LA, Malli A, Chen W, Mumtaz K. Acute liver failure due to liver parenchymal infiltration with acute myelogenous leukaemia in a patient with myelodysplastic syndrome. BMJ Case Rep. 2018 Jun 28;2018:bcr2018224590. doi: 10.1136/bcr2018-224590.

2. Goor Y, Goor O, Michalewitcz R, Cabili S. Acute myeloid leukemia presenting as obstructive jaundice. J Clin Gastroenterol. 2002 Apr;34(4):4856. doi: 10.1097/00004836-200204000-00023.

3. Mathews E, Laurie T, O'Riordan K, Nabhan C. Liver involvement with acute myeloid leukemia. Case Rep Gastroenterol. 2008 Mar 14;2(1):121-4. doi: 10.1159/000120756.

4. Rajesh G, Sadasivan S, Hiran KR, Nandakumar R, Balakrishnan V. Acute myeloid leukemia presenting as obstructive jaundice. Indian J Gastroenterol. 2006 Mar-Apr;25(2):93-4.

5. Phipps MM, Barraza LH, LaSota ED, Sobieszczyk ME, Pereira MR, Zheng EX, Fox AN, Zucker J, Verna EC. Acute Liver Injury in COVID-19: 


\section{Acute myelogenous leukemia and COVID-19 infection}

Eur J Transl Myol 31 (3): 9817, 2021 doi: 10.4081/ejtm.2021.9817

Prevalence and Association with Clinical Outcomes in a Large U.S. Cohort. Hepatology. 2020 Sep;72(3):807-817. doi: 10.1002/hep.31404.

6. Sun J, Aghemo A, Forner A, Valenti L. COVID-19 and liver disease. Liver Int. 2020 Jun;40(6):12781281. doi: 10.1111/liv.14470.
7. Bernal W, Wendon J. Acute liver failure. N Engl J Med. 2013 Dec 26;369(26):2525-34. doi: 10.1056/NEJMra1208937.

Submitted: April 18, 2021

Revision received: May 18, 2021 Accepted for publication: May 22, 2021 